\title{
Intelligent Car Control for a Smart Car
}

\author{
Md. Nazmul Hasan \\ Department of CSE, \\ Stamford University Bangladesh \\ 51 Siddeswari, Dhaka, Bangladesh
}

\author{
S. M Didar-Al- Alam \\ Department of CIT, \\ Islamic University of Technology \\ Boardbazar,Gazipur, Bangladesh
}

\author{
Sikder Rezwanul Huq \\ Department of CSE, \\ Stamford University Bangladesh \\ 51 Siddeswari, Dhaka, Bangladesh
}

\begin{abstract}
From the beginning of the artificial intelligence there was a desire of having a fully automated intelligent car. Numbers of experiments have been done and some of them were very much fruitful. As a result now we have intelligent smart cars. These cars are intelligent and can take some of the decision of their own. But they actually assist the driver for a limited amount of time. None of them are fully automated. We think a fully automated transportation can only be possible by having a combination of intelligent car and traffic system as well the environment like road on which the car actually moves. In this paper we have tried to discuss a new idea for an autonomous transportation system- a complete solution.
\end{abstract}

\section{General Terms}

Artificial Intelligence, Intelligent Car

\section{Keywords}

Intelligent Transportation, GPS (Global Positioning System), ACC (Adaptive Cruise Control), AHS (Automated Highway Systems), Smart Cars, DLCC (Dynamic Laser Cruise Control).

\section{INTRODUCTION}

Autonomous or Adaptive Cruise Control (ACC) is mostly used intelligent system for smart car controlling. Normally a radar or laser setup is used that allows the vehicle to slow when the front vehicle is slowing down and speed up to the preset speed that the traffic allows when no vehicle is in front. But these systems can only take control or take decision in straight roads where there is no possibility of taking decision about changing the route. But in this paper we tried to offer a system when it is fully autonomous. Here we have not only considered the intelligence in the car but also in the traffic system, so that an intelligent network can be established which is able to take decision for end to end transportation without any human interaction.

\section{THE CHALLENGE}

There are basically two challenges related with this intelligent transportation system. Those are -

\section{Technical Challenge \\ 2. Social Challenge}

As technical challenge we face problems in designing the sensors and the control systems and also problem in source to destination decisions. On the other hand as a Social Challenge we found the problems like- getting people to trust into automated car, getting legislators permission for being car onto the public roads, and untangling the legal issues of liability for any mishaps with no person in charge. Now the solutions to get a automated car can be broken into four sub-systems like-

1. Sensing: knowing where an obstacle is and what is around it which means sensing the surrounding.

2. Navigation: getting to the target location from the present location.

3. Motion planning: Moving on the road, avoiding obstacles, avoiding harm to the people, and obey to the rules of the road which means actually taking decision of breaking, accelerating, turning etc.

4. Control of the vehicle itself: actuating the system's decisions.

\section{AVAILABLE SYSTEMS}

Typically for ACC most popular systems are-

1. Laser Based ACC

2. Radar Based ACC

Presently the laser based ACC which is low cost but available. It is available in near luxury cars within 400-600 USD/euro. But the problem with this is it fails to detect and track vehicle well in adverse weather conditions. These cars are also unable to track dirty non reflective vehicles. Laser-based sensors have to be exposed and typically found in the lower grill offset. On the other hand Radar based ACC gives better performance then laser based ACC sacrificing more cost. They are available in luxury cars within 1000-3000 USD/euro. Available systems basically focus on automatic speed adaptation technique and intelligent breaking. They don't bother about route choosing and track changing. Normally they follow the front car and maintain safe distance from that car in order to take speed adaptation decisions. Here is a figure that shows how it is been done. Jaguar Cars and Mercedes-Benz first offered radar-based systems in 1999. Mercedes-Benz offered the 'Distronic Plus' system that can halt the car if necessary on their S-Class luxury sedans in 2006. Audi Q7 also offers this feature. Toyota's

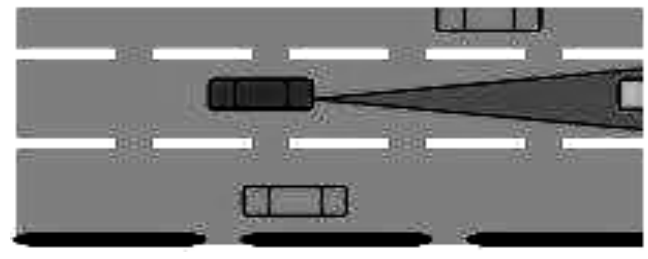

Figure 1: Adaptive Cruise Control (ACC)

Lexus first brought laser-based systems to the US market with the 2001 Lexus LS430 "DLCC" (Dynamic Laser Cruise 
Control). It also offered radar-based systems on its IS, ES, GS and LS models and the laser based on its RX model. Acura RL features Collision Mitigation Braking System. It can alert drivers of objects if the distance is less. It brakes slightly and tugs at the seatbelts too. Even if the driver doesn't react then the RL retracts, locks the seatbelts and brakes hard. Lexus LS430/460 also offers the system.

\section{PAST RESEARCHES}

Different Research groups did researches on different issues related to the system. Among them some were very fruitful that showed new pathways. Automated highway systems (AHS) are an effort to construct special lanes on existing highways that would be equipped with magnets or other infrastructure to allow vehicles to stay in the center of the lane, while communicating with other vehicles (and with a central system) to avoid collision and manage traffic. AHS allows specially equipped cars to join the system using special 'acceleration lanes' and to leave through 'deceleration lanes'. When leaving the system each car verifies that its driver is ready to take control of the vehicle, and if that is not the case, the system parks the car safely in an area. The implementation of the AHS demo is done in 1997 near San Diego, sponsored by the US government, in coordination with the State of California and Carnegie Mellon University. The test site is a 12-kilometer, high-occupancy-vehicle (HOV) segment of Interstate 15, 16 kilometers north of downtown San Diego. The event generated much press coverage which was a great achievement. The technology is become the subject of a book. This united effort by the US government seems to have been pretty much abandoned because of social and political forces, above all else the desire to create a less futuristic and more marketable solution. As in 2007, a three-year project is underway to allow robot controlled vehicles, including buses and trucks, to use a special lane along 20 Interstate 805 . The intention is to allow the vehicles to travel at shorter following distances and thereby allow more vehicles to use the lanes. The vehicles will still have drivers since they need to enter and exit the special lanes. Before entering those lanes car should have take help from the driver who starts up the car, speed up into a certain limit, enter into a safe state as well as a safe lane and then take over the control of the car into itself. Here car is half automated where car needs some assistant from the human as before being automated. The system is being designed by Swoop Technology, based in San Diego country. Over here we have analyzed the past research which was reached up to a level of assisting driver for taking decision while driving but still it needs most of the involvement of the drivers. Those researches show the pathway of the automated car.

\section{OUR CONTRIBUTION}

\subsection{Identifying the basic things that need to be autonomous}

In order to drive a car, a system would need to:

1. Understand its environment (Sensors)

2. Identify where it is and where it wants to go (Navigation)

3. Identify its way in the traffic (Motion planning)

4. Operate the staring of the vehicle (Actuation)
It is concluded that almost $2 \frac{1}{2}$ of these problems are already solved which varies in terms of environment and place. Navigation and Actuation problem solved completely, and Sensors partially, but improving fast. The main unsolved part is the motion planning which is our main concern.

\subsubsection{Sensors}

Sensors employed in driverless cars vary from the minimalist ARGO project's monochrome stereoscopy to Mobileye's intermodal (video, infra-red, laser, radar) approach. The minimalist approach imitates the human situation most closely, while the multi-modal approach is "greedy" in the sense that it seeks to obtain as much information as is possible by current technology, even at the occasional cost of one car's detection system interfering with another's thus create another problem.

\subsubsection{Navigation}

The ability to plot a route from where the vehicle is to where the user wants to be has been available for several years. These systems, based on the US military's Global Positioning System are now available as standard car fittings, and use satellite transmissions to ascertain the current location, and an on-board street database to derive a route to the target. The more sophisticated systems also receive radio updates on road blockages, and adapt accordingly. There are also sensors that greatly affect the whole nature of it.

\subsubsection{Control of vehicle}

As automotive technology matures, more and more functions of the underlying engine, gearbox etc. are no longer directly controlled by the driver by mechanical means, but rather via a computer, which receives instructions from the driver as inputs and delivers the desired effect by means of electronic throttle control, and other drive-by-wire elements. Therefore, the technology for a computer to control all aspects of a vehicle is well understood.

\subsection{Some things which assists a human as well a automated car}

These systems warn or inform the driver about events that may have passed unnoticed, such as

- $\quad$ Lane Departure Warning System (LDWS)

- Visibility aids for the driver, to cover blind spots and enhanced vision systems such as radar

- Wireless vehicle safety communications and night vision.

- Infrastructure-based, driver warning/informationgiving systems.

\section{LATEST ACHIEVEMENT}

Google modified Toyota prius [6] uses an array of sensors to navigate a public road without human involvement. It includes different types of sensors like -

- GPS: The Global Positioning System (GPS) [1] is a spacebased global navigation satellite system that provides reliable location and time information in all weather and at all times and anywhere on or near the Earth when and where there is an unobstructed line of sight to four or more GPS satellites. 
- Motion Sensor: This one is used for monitoring the speed of the car.

- LIDAR: A rotating sensor on the roof which scans around

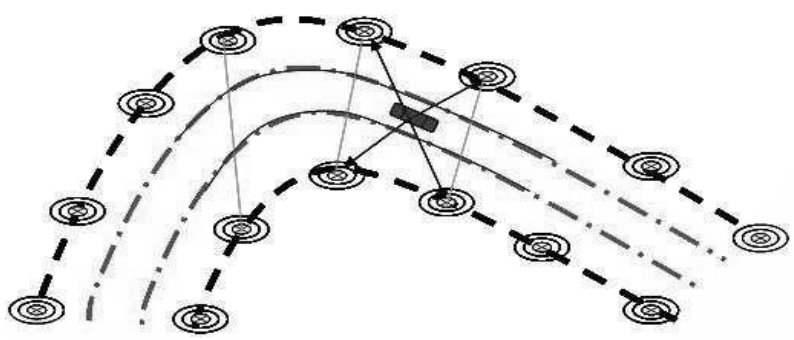

Figure 2: Autonomous Speed Adaptation with Intelligent Highway

200 feet in all the direction of the car to generate a precise three dimensional map on the car's surrounding.

- Position estimator: A sensor mounted on the left rear wheel which measures the small movement made by the car and to accurately locate its position on the map.

- Video Camera: A camera near the rear view mirror deflects traffic lights and helps the car's onboard computer recognize moving obstacle like pedestrian and bicyclists.

- Radar: Four standard automotive radar sensors, three in front and one in the rear, help to determine the position of the distant objects.

By using these kind of sensors this car overcome the problem of Navigating and actuating itself, hence using various types of sensor it also achieved good sensing capability but still motion planning is a challenging issue for that car on which factor basically we emphasizes.

\section{OUR PROPOSAL}

Here in this paper we are actually trying to offer a complete end to end solution we have divided our proposal into five specific parts:
1. Route Analysis
2. Autonomous Speed Adaptation
3. Lane Detection \& Change
4. Intelligent Breaking System
5. Artificial vision image processing
6. Avoid Collusion
7. Security Issues

\subsection{Route Analysis}

When we are starting with our car we need to fix our route towards our destination. Now with an intelligent system we only need to specify our destination. From the GPS [1] the car knows its present position and from the digital map it knows all the routes towards the destination. It then finds out all alternatives of the routes and gives the best low cost and low distance solution. It also gives an option that from the possible solution which one the owner would like to have. If he gives a choice it follows the gives route. Else it would follow the low cost route or the optimum solution. This is the first step of the journey where the route is already been fixed. Now the car has to move towards the destination on the route it has selected.

\subsection{Autonomous Speed Adaptation}

This is actually a well recognized system that is already in use But we are offering a few modifications on the present system Causes for these modifications are described here. In the present system the speed adaptation [2] is basically done depending on the front vehicle. If the vehicle slows down the car slows its speed and if the car accelerate then the car accelerates. Here the driver has to set the speed limit that up to which speed it can accelerate and also have to be concerned about the signals. That means though we are actually saying that it is automatic but much of the decisions are given by the driver. Again when there is no vehicle in front then it can not take any decision depending on the signal that the red light is on and it needs to be slow down. We offer that the previous adaptation system would be in use but in addition to it the highway should be made intelligent also. In the signal positions the signal posts should communicate with all the vehicles that already a red signal has been shown so slow down or green light is on you can move. When ever the car is entering any speed zone the speed limit posts should inform the cars that this is a speed limit area so the maximum speed limit should be that. So the car can itself take the maximum speed decision. In this way two of the human dependency can be reduced.

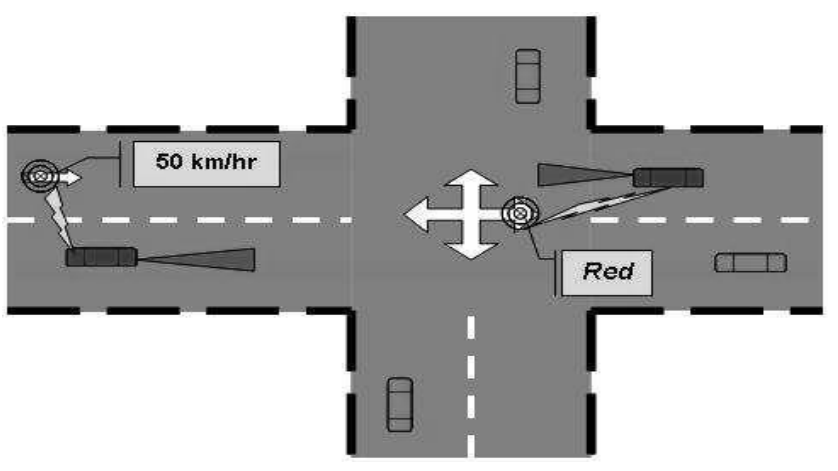

Fig. 3: Lane Detection

\subsection{Lane Detection and Change}

Lane detection is a major concern in order to move automatically on the road. From the GPS [1] we know that where there is a curve on the road but how much bend is there it is not clear from the GPS or the digital map [1]. Again from the Radar or Laser sensor it is possible to get a view that there is curve if obstacle is there though it is tough to have a correct estimation about the bend and even sometimes hard to notify the curve. But if we can estimate that in which lane we are now and always keep our car on the track then with the bend of the lane the car will also take a turn itself. So no problem arises with taking a decision in a curve road. That makes the automation possible in any kind of road not only in straight ones. Now this lane detection can be achieved by having roadside wireless signals. These signals will always transmit that where is it residing and how many lanes are there in the road. From these readings the car knows that in which lane he is now. And by measuring the distance it ensures that it resides always in the same lane. Again lane changing is also a difficult decision to take. In present smart cars we have to change the lane manually. So for the time human interaction is needed. This makes actually the whole automation useless as the driver has to be concern always about the change of the lane. But this 
can be made automated using two more radar or sensor device in the car. The figure shows that how this can be done. Two laser or radar devices residing on the sides will always search that is there any vehicle moving either of the sides. Another laser or radar device on the back checks that is there any vehicle trying to overtake. If it finds on the possibilities negative it takes the decision for having a lane change. This way the lane

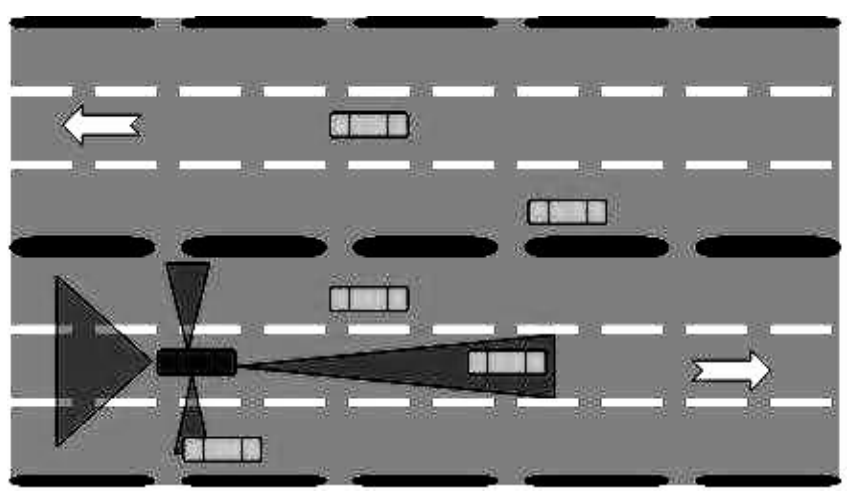

Figure 4: Lane Changing Decision

changing can be made automated. But using three more devices is a costly choice. So we can perform the task using one device. This will have time scheduled checking on three of the sides and gives the same output that we want to have.

\subsection{Intelligent Breaking System}

As speed adaptation is needed thus is the speed control. So we need to be concerned about the breaking system. Now the breaking system mostly depends on finding any obstacles. If it finds any obstacle it would slow down. It would also measure its speed and size. Now that requires a video camera to take effect. Now this analysis result helps the car to take decision that when and how to break. It first slows itself down gradually and when it finds a break situation it breaks with some more additional tasks. It should tight the seatbelt to ensure the safety of the passengers, give horn to alert others and in an extreme situation it should give a signal to the driver to notify him about the situation.

\subsection{Artificial vision image processing}

This one is the feather which could be achieved by image processing. Actual automated system could be achieved only when we incorporate our car with a sensor which can sense like human \& take decision like human. In GPS we get data which is not precisely instant. Other sensor that we use can sense the things or obstacles in front of our car but for getting instant feedback we need image processing software which gets image from the Video Camera. After processing that our onboard computer takes necessary steps for motion control of the automated car. This image processing basically helps us for avoid the accident in case of sudden obstacles come in front of the automated car which is basically acts like human intelligence.

\subsection{Avoid Collusion}

The cars will avoid collisions with obstacles located in the environment using laser (long range) and ultrasonic (shortrange) sensors. Laser will be used for identifying the obstacles which is in front of that car. On the other hand short range sensor like ultra sonic sensor will be used for avoiding the collusion of the adjacent lane cars or been struck the road divider. Road divider will also have some short range sensor which will be sensed by the car when it crosses the certain threshold limit of the distance between the car and the divider.

\subsection{Assistance in Turning}

All of the intelligent that have already developed is well suited for the straight road. Some of the statistics shows that around $90 \%$ time car is autonomous that means in that time car was independent of driver though he was present; rest of the $10 \%$ time those intelligent cars needs the assistant of the drivers. It is true that that $10 \%$ time was the situation when the car needs to take left or right turn as because then more intelligent decision should have to take by the car which is not achieved by the intelligent car yet. Over this situation our proposal is to use ultra sonic senor on the divider of the road which will be sensed by the car. By using the intensity of that sensor output our car will know how closer he is to the divider. When this distance is tends to be shorter then it takes necessary steps for making that distance constant, like take left turn when divider is on right or vice versa. Thus we can achieve the problem of left and right turning. On the meantime cars ultra sonic sensor is used for detecting the adjacent lanes cars position to avoid the collusion with those car.

\subsection{Security}

Different security issues should be maintained with intelligence. This can be done by applying different systems. Our proposed systems are-

a. The starting should be done as a button press and that button should have a finger print analysis system. The owner and the user of the car all have their finger prints prerecorded. This ensures that no other person is able to start the car by any illegal mean.

b. Airbags [5] should be controlled centrally and the car should have the decision taking capability that within what time they should be exposed depending on the speed of the cars and the force of the collision.

c. The car should always have communication with the central traffic system so that at any mishap it can inform the central system. The central system should always track the vehicle. This ensures help on the spot at any case of emergency.

\section{ADVANTAGES \& DISADVANTAGES}

The benefits we are expecting to have are as follows:

1. It would be fully autonomous system from end to end without any human interaction. So it gives a full relax in driving that means the driverless transportation would be possible. 
2. The intelligent car is fully aware of its own condition. So there is no need to be concerned about the condition of the vehicle. It is checked automatically and problem can be detected and fixed from the central system.

3. The security issues makes the automated car a much safer driving experience. It is also aware of the conditions of the passengers and at any disturbance with their health condition and at any emergency situation it can inform the central system and take necessary actions.

4. We expect to have a much lower rate of accidents as automation will add more accuracy in driving. Moreover the automated driving will not depend on the mental condition of the driver. So no hamper would be there.

5. Customization would become an easy task as with a single soft copy of a module we can define the full customization of the vehicle. The car recognizes the user and it itself makes the customizations.

The basic drawback of this proposal would face is the cost. The devices we are about to use here will make the cost increase. But it should be acceptable as a new technology always comes a bit costly. Moreover the car is offering no driver and diagnosis needed that saves money. So in spite of its cost hopefully it would be a fruitful proposal to be implemented in near future.

\section{CONCLUSION}

This proposal gives a view to a complete automation of the transportation system. But further works should be done to find out its faults and to solve them. But as far as we understood we think that it can give a complete guideline for the system and should be very much helpful for further researches. We think that with a full automated transportation the dream of having the automated society will come to true. Automated transportation will be one major step in that dream come true. By using multiple sensors effectively we can achieve the automated car, which will relief the people of taking burden of driving a car that saves thousand of working hour of human. We believe if our proposal in incorporated with the recent advancement then with 5 year we will find the fully self dependent automated car. Over here is a factor of cost as for getting one automated car people have to spend more but that will be in return when they can save thousands of working hours as well as get safe, easy, comfortable, optimum and reliable journey.

\section{REFERENCES}

[1] Basnayake C; Mezentsev O; Lachapelle G; Cannon M (2004): "A Portable Vehicular Navigation System Using High Sensitivity GPS Augmented with Inertial Sensors and MapMatching", SAE Paper 2004-01-0748.
[2] Carsten O ; Tate F (2005): "Intelligent Speed Adaptation: Accident Savings and Cost-Benefit Analysis",

Accident Analysis and Prevention 37, pp.407-416.

[3] ETSC (2006): "Intelligent Speed Assistance Myths and Reality: ETSC Position on ISA", European Transport Safety Council, May 2006

[4] Regan M; Triggs T; Young K; Tomasevic N; Mitsopoulos E; Stephan K ; Tingvall C (2006) :"On-road Evaluation of ISA, Following Distance Warning and Seat Belt Reminder Systems: Final Results of the TAC Safecar Project", Monash University Accident Research Centre, September 2006.

[5] Carsten O (2001) : "ISA: the Best Collision Avoidance System?", Proceedings of 17th Conference on the Enhanced Safety of Vehicles, Netherlands.

\section{AUTHORS}

Md. Nazmul Hasan has completed his graduation from Islamic University of Technology (IUT) in November 2008 After his graduation he has joined in Stamford University Bangladesh as a lecturer of Computer Science department and till now he is serving here. He is also been working as a part time faculty member of Islamic University of Technology (IUT). His research interest includes Distributed Systems, Parallel Processing, Sensor Network, Cellular Network, Artificial Intelligence etc.

S. M. Didar-Al-Alam has completed his graduation form Islamic University of Technology (IUT) in November 2008. Just after his graduation he joined Islamic University of Technology (IUT) as a Lecturer in Department of Computer Science and Information Technology (CIT) and till now he is serving IUT. His major research interests are related to the field of Artificial Intelligence, Wireless Communication \& Networking, Ubiquitous Computing.

Sikder Rezwanul Huq has completed his graduation from Islamic University of Technology (IUT) in November 2009. After his graduation he has joined at Stamford University Bangladesh as lecturer of Computer Science department and working to date. His research interest includes Grid Computing, Distributed Systems, Parallel Processing, Sensor Network, Artificial Intelligence etc. 